\title{
Evaluation of the efficacy and safety of flumazenil in the treatment of portal systemic encephalopathy: a double blind, randomised, placebo controlled multicentre study
}

\author{
K Gyr, R Meier, J Häussler, P Boulétreau, W E Fleig, A Gatta, A Holstege, \\ G Pomier-Layrargues, S W Schalm, M Groeneweg, G Scollo-Lavizzari, \\ E Ventura, M L Zeneroli, Roger Williams, Y Yoo, R Amrein
}

Correspondence to: Professor K Gyr, Department Innere Medizin, Medizinische UniversitatsPoliklinik, Kantonsspital Basel, Petersgraben 4, CH 4031, Basel, Switzerland. Accepted for publication 11 March 1996

\begin{abstract}
Background-Portal systemic encephalopathy (PSE) is a complex neuropsychiatric syndrome associated with hepatic failure. Small scale studies have shown the benzodiazepine receptor antagonist flumazenil to be effective in ameliorating PSE.

Aims-To determine the efficacy of flumazenil in patients with non-comatous mild to moderate PSE (stages I to III) due to severe chronic liver disease.

Patients-49 male and female adults without symptoms of severe bleeding and sepsis and who screened negative for benzodiazepine in both blood and urine, were included in the study.

Methods-Patients were randomised to receive either three sequential bolus injections of flumazenil $(0.4,0.8$, and $1 \mathrm{mg})$ or placebo at one minute intervals, followed by intravenous infusions of either flumazenil $(1 \mathrm{mg} / \mathrm{h})$ or placebo for three hours. Clinical PSE grading and vital signs were assessed hourly during baseline and post-treatment periods and half hourly during treatment. The main outcome measures were improvement in group average PSE score and reduction of two points in individual PSE score (clinically relevant improvement).

Results-The mean average improvement in the PSE score in the subjects treated with flumazenil was not statistically significantly different from placebo. However, for patients showing clinically relevant improvement, the difference between flumazenil and placebo was statistically significant (seven of $28 v$ none of $21 ; p=0 \cdot 015)$. Flumazenil was well tolerated.

Conclusions-A subgroup of patients with PSE resulting from chronic liver disease may benefit from the administration of flumazenil.

(Gut 1996; 39: 319-324)
\end{abstract}

Keywords: portal systemic encephalopathy, PSE, liver disease, flumazenil, benzodiazepines.

Portal systemic encephalopathy (PSE) is a complex neuropsychiatric syndrome associated with hepatic failure, which is characterised by central nervous system depression and an increase in portal systemic shunting. ${ }^{1}$ In clinical practice the severity of PSE can be graded into four stages (I to IV) based on the mental state and neuromuscular function of the patient using criteria such as sleep disorders, disorientation, somnolence, stupor, and coma. ${ }^{23}$ Animal studies have shown that antagonists of $\gamma$-aminobutyric acid and benzodiazepines can attenuate the increased neural inhibition associated with PSE. ${ }^{4}$ Flumazenil (Ro 151788), the first competitive benzodiazepine receptor antagonist, has a high affinity for the benzodiazepine receptor, and rapidly reverses the hypnotic-sedative effects of benzodiazepines following intravenous administration. ${ }^{56}$ Anecdotal clinical observations ${ }^{7}$ suggesting that flumazenil may be effective in ameliorating symptoms of PSE in patients with chronic liver disease have been confirmed in several small scale open and three double blind clinical studies. ${ }^{8-13}$ One of the latter studies has been published in abstract form only. ${ }^{11}$ These findings are consistent with the endogenous benzodiazepine theory, which has been extensively reviewed. ${ }^{114}$ Nevertheless opposite results have been published ${ }^{15}$ and considerable confusion exists as to the true efficacy of flumazenil. This double blind study was conducted to evaluate the acute therapeutic effect of intravenous flumazenil on the clinical severity of PSE in a group of patients with non-comatous mild to moderate PSE (stage I to III) who were carefully screened negative for benzodiazepine concentrations in both blood and urine. To obtain a sufficient number of patients while maintaining stringent entry criteria, the trial was designed as an international multicentre study.

\section{Methods}

\section{Patients and study design}

The trial protocol was approved by each local review board ethical committee and all patients or their relatives gave informed consent to participate.

To establish stable underlying clinical conditions and to avoid influence from factors such as cerebral oedema (for example, in hepatic coma (stage IV)), the patient inclusion 
1 Anamnestic

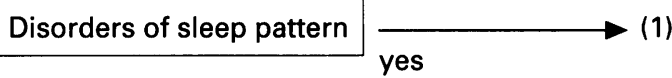

2 Clinical status

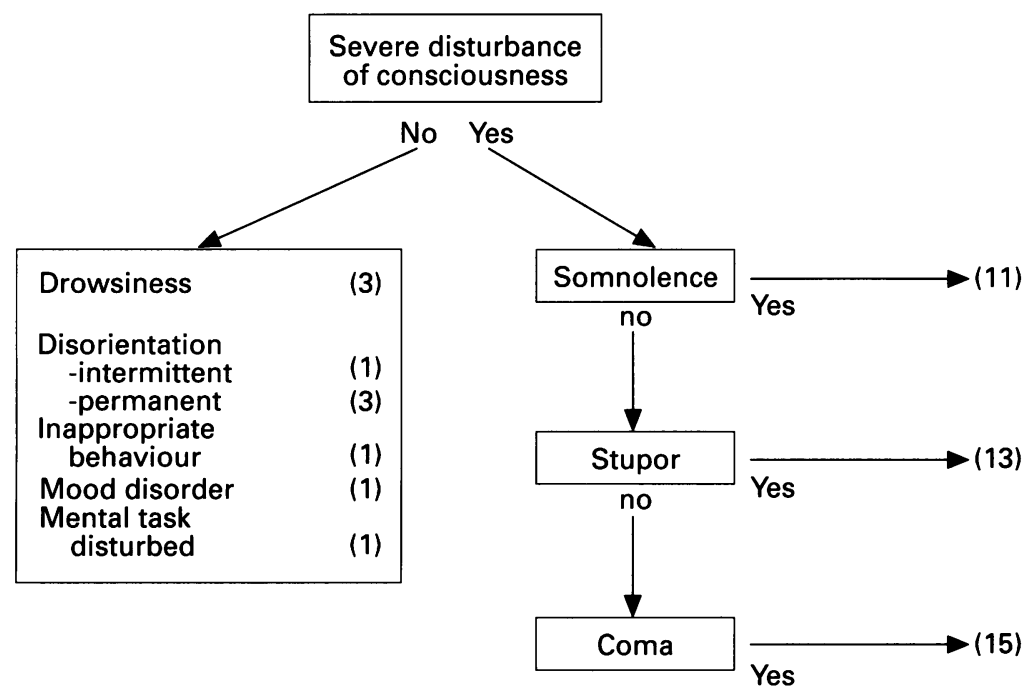

\begin{tabular}{|ll|}
\hline PSE & Sum score \\
STAGE 0 & $<3$ \\
STAGE I & $3-4$ \\
STAGE II & $5-10$ \\
STAGE III & $11-14$ \\
STAGE IV & $15-16$ \\
\hline
\end{tabular}

Figure 1: Clinical PSE score. Sum score of anamnestic and clinical items representing clinical severity of PSE.
(4) Coma (unarousability, no or unlocalised motor reactions to painful stimuli).

The score items were weighted so that major disturbances of consciousness (PSE stage III and IV) were associated with scores of 11 or higher. PSE stage II was defined to range from scores of 5 to 10 and stage I from 3 to 4 . PSE was also categorised according to electroencephalogram (EEG) characteristics, based on a combination of visual inspection or automated EEG analysis, or both, by one central assessor (G Scollo-Lavizzari). ${ }^{16} 17$ This evaluation was done under double blind conditions and the assessor was not aware of the clinical outcome.

Care was taken to exclude PSE episodes resulting from precipitating clinical conditions that could interfere with the effect of flumazenil on PSE. Consequently PSE episodes resulting from common precipitating situations such as severe bleeding and infection (for example, sepsis) were excluded resulting in a selection of patients with apparently more spontaneous and stable PSE in chronic liver disease.

Furthermore, patients with acute fulminant liver failure, coma (PSE/stage IV) at any point of the study, metabolic coma other than due to liver failure, hepatitis superimposed on cirrhosis, liver tumours, severe cerebral atrophy as assessed by cranial computer aided tomography, and psychiatric disease except PSE as well as patients who reported to have taken psychotropic medication (including benzodiazepines) were excluded from the study. In addition, screening tests in blood and urine samples for opiates, barbiturates, and benzodiazepines (Abbott $\mathrm{TD}_{\mathrm{X}}$, Abbott Laboratories, Chicago, IL, fluorescence-polarisation immunoassay, with a detection limit $10-100 \mathrm{ng} / \mathrm{ml}$ in blood, $\leqslant 200 \mathrm{ng} / \mathrm{ml}$ in urine) were performed during the baseline period.

As the sensitivity and specificity of the screening test is limited, the biological material was frozen and afterwards analysed by a central laboratory for benzodiazepines using a sensitive high pressure liquid chromatography (HPLC) method (sensitivity: $\leqslant 50 \mathrm{ng} / \mathrm{ml}$ in blood and $10-100 \mathrm{ng} / \mathrm{ml}$ in urine). Patients were randomised and the treatment was started before the results of the screening test were available. Patients are therefore included in the intent to treat analysis independent of the fact that they had a positive benzodiazepine test or not, but patients with a positive benzodiazepine test were excluded from the per protocol analysis.

The severity of the underlying liver disease was assessed according to the Child-Pugh grading system. ${ }^{18}$

The study was 12 hours in duration and consisted of three periods: a four hour baseline observation period, a three hour double blind treatment period, and a five hour post-treatment observation period. After the baseline observation period, patients were randomised to receive (at one minute intervals) three sequential bolus injections of flumazenil $(0.4$, 0.8 , and $1 \mathrm{mg}$ ) or placebo, after which patients were given either intravenous infusions of either flumazenil $(1 \mathrm{mg} / \mathrm{h})$ or placebo for tions of sevens), mood disorder, inappropriate behaviour.

(2) Somnolence (arousable to physical stimuli such as mild prodding or shaking only). pain).
(3) Stupor (localised motor response to 
TABLE I Patients excluded from per protocol analysis

\begin{tabular}{lcc}
\hline $\begin{array}{l}\text { Protocol deviations (exclusion from } \\
\text { standard analysis) }\end{array}$ & Flumazenil & Placebo \\
\hline Positive benzodiazepine screening & 3 & 1 \\
Missing or inadequate benzodiazepine & & \\
screening & 8 & 4 \\
Liver tumour & 2 & 1 \\
Severe cerebral, atrophy & 0 & 1 \\
Meal during baseline & 0 & 2 \\
Incomplete assessment of PSE score & 1 & 10 \\
\hline Total & 14 & \\
\hline
\end{tabular}

three hours. Only the following additional treatments were permitted: saline, glucose, lactulose, potassium, and vitamin $\mathrm{K}$.

Clinical PSE grading was performed at 60 minute intervals during the baseline and post-treatment periods and every 30 minutes during treatment. The first assessment took place within five minutes of the bolus injections. At each time point, vital signs and rectal body temperature were recorded. EEG recordings were made continuously during the first and the last 20 minutes of each period.

Response was defined as improvement in the average PSE score (mean of all individual scores) during treatment and post-treatment compared with baseline, whereas a clinically relevant improvement was defined as a two point improvement in PSE score at any time during treatment compared with baseline.
Clinical PSE scores and EEG grades were compared both regarding severity and improvement.

Clinical experience with flumazenil has shown that its onset of action is generally very rapid. ${ }^{6}$ As these rapid changes could be blurred by focusing on average scores, a separate assessment of the changes between the time points immediately before (last baseline score) and immediately after the fractionated bolus administration of flumazenil (first treatment score) was performed.

Safety evaluations included registration of adverse events and detailed blood laboratory investigations at baseline and at the end of the post-treatment observation period.

\section{Statistical analyses}

Statistical analyses were performed on two different patient populations. All patients randomised with at least one on drug observation constitute the intent to treat population, whereas patients deviating from protocol were excluded from the evaluation of the per protocol population. Clinical response data were analysed using a rank test for independent samples (Wilcoxon two sample test with continuity correction of 0.5$),{ }^{18}$ while the numbers of patients in both treatment groups showing clinically relevant improvement were compared using Fisher's exact test.

TABLE II Demographic and clinical data of treatment groups

\begin{tabular}{|c|c|c|c|c|}
\hline & \multicolumn{2}{|c|}{ All patients (ITT) } & \multicolumn{2}{|c|}{ Per protocol analysis } \\
\hline & Flumazenil & Placebo & Flumazenil & Placebo \\
\hline Number & 28 & 21 & 14 & 11 \\
\hline \multicolumn{5}{|l|}{$\operatorname{Sex}(n)$} \\
\hline Male & 22 & 12 & 11 & 6 \\
\hline Female & 6 & 9 & 3 & \\
\hline Age $(y)$ mean $(S D)$ & $55 \cdot 5(9 \cdot 4)$ & $53 \cdot 6(10 \cdot 3)$ & $56 \cdot 9(7 \cdot 8)$ & $52 \cdot 2(9)$ \\
\hline Baseline PSE score mean (SD) & $7 \cdot 3(3 \cdot 5)$ & $7 \cdot 5(4 \cdot 1)$ & $7 \cdot 1(4)$ & $6 \cdot 8(4 \cdot 5)$ \\
\hline \multicolumn{5}{|l|}{ Baseline PSE stage (n) } \\
\hline I & 7 & 6 & 6 & 5 \\
\hline II & 14 & 8 & 4 & 3 \\
\hline III & 7 & 7 & 4 & 3 \\
\hline \multicolumn{5}{|l|}{ Child-Pugh score (n) } \\
\hline A & 1 & 1 & 0 & 1 \\
\hline B & 7 & 6 & 4 & 4 \\
\hline $\mathrm{C}$ & 20 & 14 & 10 & 6 \\
\hline \multicolumn{5}{|l|}{ Disease aetiology } \\
\hline Alcoholic & 15 & 10 & 8 & 3 \\
\hline Liver tumour & 1 & 0 & 0 & 0 \\
\hline HBV & 6 & 7 & 4 & 5 \\
\hline $\mathrm{HCV}$ & 1 & 1 & 1 & 1 \\
\hline NANB & 1 & 0 & 0 & 0 \\
\hline Autoimmune chronic hepatitis & 0 & 1 & 0 & 1 \\
\hline Portal vein thrombosis & 1 & 1 & 0 & 0 \\
\hline Schistsomiasis & 1 & 0 & 0 & 0 \\
\hline Unknown & 2 & 1 & 1 & 1 \\
\hline
\end{tabular}

ITT=intent to treat.

TABLE III Number of patients with clinically relevant response (improvement of at least two points in PSE score) in per protocol and intent to treat analysis

\begin{tabular}{|c|c|c|c|c|c|c|}
\hline & \multicolumn{3}{|c|}{ Per protocol analysis } & \multicolumn{3}{|c|}{ Intent to treat analysis } \\
\hline & Flumazenil & Placebo & Total & Flumazenil & Placebo & Total \\
\hline $\begin{array}{l}\text { Improved } \\
\text { Not improved }\end{array}$ & $\begin{array}{l}5 \\
9\end{array}$ & $\begin{array}{r}0 \\
11\end{array}$ & $\begin{array}{r}5 \\
20\end{array}$ & $\begin{array}{r}7 \\
21\end{array}$ & $\begin{array}{r}0 \\
21\end{array}$ & $\begin{array}{c}7 \\
42\end{array}$ \\
\hline \multirow[t]{2}{*}{ Total } & 14 & 11 & 25 & 28 & 21 & 49 \\
\hline & \multicolumn{3}{|c|}{$\mathrm{p}=0.046$} & \multicolumn{3}{|c|}{$p=0.015$} \\
\hline
\end{tabular}

$\star$ Fisher's exact test (two tailed) for comparison between flumazenil and placebo.

\section{Results}

Of the 49 patients enrolled and constituting the intent to treat sample, 24 patients did not comply with the strict inclusion criteria leaving 25 patients in the per protocol sample. The most frequent reason for exclusion from the per protocol population was of technical nature. For 12 patients, the frozen plasma and urine samples were lost during transportation to Switzerland. The presence of benzodiazepines at measurable concentrations in plasma or urine could therefore not be excluded in these patients. Other reasons were positive benzodiazepine screening, liver tumour, severe cerebral atrophy, and incomplete PSE scoring (Table I). Table II gives the demographic data, Child-Pugh scores, PSE stages, and mean baseline PSE scores for the intent to treat and per protocol population. No significant difference was found between any of the treatment groups for any demographic, clinical variable or exclusion criteria. Alcoholism and hepatitis B were the most frequent reasons for the underlying liver disease. One patient in the flumazenil group had a portocaval shunt. Six patients in the flumazenil group and four patients in the placebo group had haemoglobin values below $10 \mathrm{~g} \%$ but no bleeding was detected at hospital admission.

Improvement was higher in the flumazenil group than in the placebo group in terms of mean PSE score. The mean improvement in flumazenil patients was 1.53 during treatment and 1.23 after treatment, compared with 0.31 


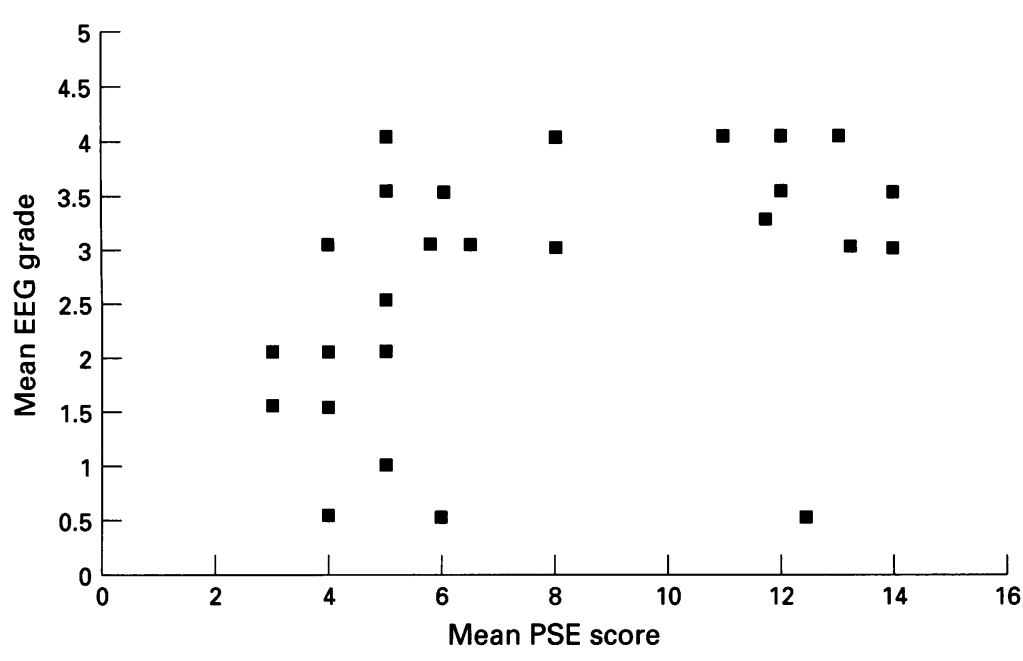

Figure 2: Relation between average baseline PSE score (mean of four measurements) and $E E G$ score (mean of two assessments) in all evaluable patients with both evaluations.

and 1.07 in the placebo group (per protocol analysis). However, the results were not statistically significant because of the low number of patients showing any change in either treatment group.

Flumazenil was significantly superior to placebo with respect to the number of patients with a clinically relevant response (Table III). Owing to the low response rate, specific predictors for a response could not be identified from the available clinical data. A clinically relevant immediate response was found in five of seven flumazenil responders in the intent to treat analysis. All five patients remained in a stable condition throughout the remaining study period. None of the other flumazenil patients and none of the placebo patients showed an immediate response.

In the intent to treat population, clinically relevant responses to flumazenil occurred in two of seven patients with PSE stage I, two of 14 with PSE stage II, and three of seven with PSE stage III. Although the overall numbers were too small to achieve statistical significance, the proportion of patients responding to flumazenil appeared to be greater in stage III compared with lower grades of PSE.

Average EEG scores calculated at baseline were compared with respective average clinical PSE scores in each patient with evaluable EEG tracings (Fig 2). The graph clearly shows that no linear relation exists between these parameters. In patients with a mild degree of PSE (PSE 55), all degrees of EEG abnormality from minor to severe were found. However, with higher degrees of clinical impairment (PSE $>5$ ), all but two patients were categorised as EEG stage III or IV.

For technical reasons, EEG recordings were obtained in only 33 patients. In view of the low numbers, it was decided not to use EEG as a separate efficacy parameter in this report.

Adverse events considered possibly or probably related to treatment occurred in four patients in the flumazenil group, the symptoms being flushing, nausea and vomiting, nausea and irritability, of which only irritability was graded as severe. There were no adverse events in the placebo group, although one patient died from respiratory failure during the course of the study. Clinical and EEG responses were not associated with any significant changes in body temperature or vital signs and deviations in laboratory parameters from baseline occurred independently of the study medication.

As expected in such severely ill patients, a total of nine deaths occurred within four weeks following the study (four flumazenil and five placebo). However, these deaths were not considered related to study medication.

\section{Discussion}

This study is, to our knowledge, the first fully published controlled randomised trial of flumazenil in patients with PSE stage I-III and was undertaken in response to somewhat inconsistent results from studies involving flumazenil in PSE associated with chronic liver disease. $^{2 ~ 8-1318}$

Patients were only included if they had chronic liver failure associated with stable mild to moderate PSE (I-III; score 3-14) thereby excluding such complicating factors as cerebral oedema. Care was taken to exclude patients in whom PSE was precipitated by severe bleeding or infection as these patients have a favourable prognosis once the causative factors have been controlled. This population was therefore inherently different from those in previous double blind studies ${ }^{11} 12$ in that a less favourable outcome would be expected.

A clinically relevant response was seen in $25-36 \%$ of patients receiving flumazenil, but in none of the placebo treated patients and the difference was statistically significant. The small number of patients showing any change in PSE score in this study prevented the group mean score becoming significant, though the mean improvement in PSE score was higher in the flumazenil group than in the placebo group. The rate of responders in this study is in keeping with that of the double blind trial by Pomier-Layrargues et $a l^{13}$ in cirrhotic patients with hepatic coma but distinctly lower than that in some uncontrolled series. ${ }^{8-10}$ In the latter studies methodological weaknesses such as the lack of a proper experimental design and the nonexclusion of patients previously exposed to benzodiazepine drugs might account for the high success rate. In this study, intake of benzodiazepines was ruled out by taking a careful medical history from each patient and appropriate search for benzodiazepines in blood and urine.

Because of the small total number of responders, subgroups of patients with PSE that may benefit from flumazenil treatment could not be determined. However, the fact that $43 \%$ of patients with PSE III responded, compared with only $14 \%$ of patients with PSE II, suggests that a clinically relevant response is more likely among more severely ill patients. These results are in line with 
those reported in patients with hepatic coma. ${ }^{13}$

Although responses to flumazenil are normally expected to occur immediately after administration, this was the case in only five of seven responders. However, it is possible that an earlier response may have been missed in the other two patients because PSE scores were not assessed until 30 and 60 minutes after drug administration. The phenomenon of a somewhat delayed response has also been seen in an uncontrolled study by Grimm et al. ${ }^{9}$

In general, EEG grading did not correlate well with PSE score, although a distinction between minor (EEG I and II) and severe (EEG III and IV) impairment could be noted in patients at a clinical PSE score of $5 / 6$ or less (that is, PSE I and II). On balance, EEG recordings may not add valuable information when studying flumazenil in advanced PSE, although this assessment may be useful in less severe stages. This view is supported by Van der Rijt et al, ${ }^{17}$ who found spectral analysis of EEG particularly useful in PSE stages I-III.

In contrast, Cadranel et $a l^{12}$ found EEG monitoring to be more rapid and more sensitive than clinical assessment in 18 PSE episodes, 12 of which were PSE III and IV. Their study population could not be compared with the patients in this study because the portal systemic encephalopathy episodes were in most cases due to sepsis and severe bleeding making the effects of flumazenil and the aggressive treatment of the underlying clinical condition more difficult to differentiate.

For the per protocol evaluation patients were excluded with unknown or positive benzodiazepine screening results despite negative history of benzodiazepine intake. Unfortunately, the very sensitive HPLC assay used in this study could not be used in 12 patients and this group represented most of the protocol violations. In most of the excluded patients, an immunoassay with lower sensitivity (Abbott $\mathrm{TD}_{\mathrm{x}}$ ) was used. While this assay would not be able to detect the recently reported plasma concentrations of 'endogenous' benzodiazepines in patients with PSE stage III and IV, 1420 it should be sensitive enough to exclude clinically relevant concentrations of exogenous benzodiazepine, which also represented the original rationale.

As the general exclusion of patients without the sensitive screening assessment could be questioned as being over-rigorous, the analysis of the intent to treat population received the same attention as the per protocol population. Presence of benzodiazepine did not seem to be an important problem in this study because only four of 49 patients had to be excluded from per protocol analysis in retrospect because of positive benzodiazepine results. It is noteworthy that two of three benzodiazepine positive patients in the flumazenil group did not respond to flumazenil, whereas the third one showed only a weak response (average improvement during treatment versus baseline 0.5 PSE points). This is in keeping with the finding of PomierLayrargues et $a l^{13}$ that the efficacy of flumazenil in PSE may not be related to the presence or absence of benzodiazepines in blood.

In conclusion, this study confirms previous data that have suggested that agonists at the benzodiazepine receptor may have a significant role in the pathogenesis of PSE. However, it remains to be determined whether these agonists are of endogenous origin or exogenous compounds that could not be detected by the assays used. Considering the sometimes critical clinical situation in these patients, the demonstrated response, together with the reported safety in this patient population, may justify the clinical use of flumazenil in patients with PSE.

Departement Innere Medizin, Kantonsspital Basel, Basel, Switzerland, K Gyr, G Scollo-Lavizzari. Departement Innere Medizin/Gastroenterologie, Kantonsspital Liestal, Liestal, Switzerland, R Meier. F Hoffmann-La Roche, Basel, Switzerland, J Häussler, R Amrein. Service d'Anesthesie Reanimation VI, Hôpital de l'Hôtel-Dieu, Lyon, France, $\mathrm{P}$ Boulétreau. First Department of Medicine, Martin Luther University, Halle/Saale, Germany, W E Fleig. Istituto di Medicina Clinica, Cattedra di Medicina Interna I, Centro di Splenoepatologia, Università di Padova, Padova, Italy, A Gatta. Klinik und Poliklinik für Innere Medizin I, Regensburg, Germany, A Holstege. André-Vallet Clinical Research Centre, Hôpital Saint-Luc, Montreal, Quebec, Canada, G Pomier-Layrargues. Academisch Ziekenhuis Dijkzigt Afd Inwendige Geneeskunde II, Rotterdam, the Netherlands, S W Schalm, M Groeneweg. Dipartimento di Medicina Interna, Clinica Medica Generale e Terapia Medica II, Università di Modena, Modena, Italy, E Ventura, M L Zeneroli. Institute of Liver Studies, King's College Hospital, London, Roger Williams. Hepatogastroenterology Branch, Department of Internal Medicine, College of Medicine, Hallym University, Kangdong, Sacred Heart Hospital, Seoul, Korea, Y Yoo

1 Basile AS, Jones EA, Skolnick P. The pathogenesis and treatment of hepatic encephalopathy: evidence for the involvement of benzodiazepine receptor ligands. Pharmacol Rev 1991; 43: 27-71.

2 Conn HO, Lieberthal $M$. The hepatic coma syndromes and lactulose. Baltimore: Williams and Wilkins, 1979: 6.

3 Jones EA, Gammal SA. Hepatic encephalopathy. In: Arias IM, Jakoby WB, Popper H, et al, eds. The liver: biology and IM, Jakoby WB, Popper H, et al, eds. The liver: biology
pathobiology. 2nd ed. New York: Raven Press, 1988.

4 Basset ML, Mullen KD, Skolnik P, Jones EA. Amelioration of hepatic encephalopathy by pharmacologic antagonism of the GABA benzodiazepine receptor complex in a rabbit model of fulminant hepatic failure. Gastroenterology 1987 93: 1069-77.

5 Haefely W. The preclinical pharmacology of flumazenil. Eur f Anaesthesiol 1988; 5 (suppl 2): 25-36.

6 Amrein R, Hetzel W, Hartmann D, Lorscheid T. Clinical pharmacology of flumazenil. Eur $\mathcal{F}$ Anaesthesiol 1988; (suppl 2): 65-80.

7 Bansky G, Meier PJ, Ziegler WH, Walser H, Schmid M, Huber M. Reversal of hepatic coma by benzodiazepine Huber M. Reversal of hepatic coma by benzodiaze

8 antagonist (Ro 15-1788). Lancet 1985; i: 1324-5. Schmid $M$. Effects of the benzodiazepine receptor Schmid M. Effects of the benzodiazepine receptor antagonist flumazenil in hepatic encep

9 Grimm G, Ferenci P, Katzenschlager R, Madl C, Schneeweiss B, Laggner AN, et al. Improvement of hepatic encephalopathy treated with flumazenil. Lance 1988; ii: $1392-4$.

10 Meier R, Gyr K. Treatment of hepatic encephalopathy with the benzodiazepine antagonist flumazenil: a pilot study. Eur f Anaesthesiol 1988; 5 (suppl 2): 139-46.

11 Frenkel AL, Hermant JL, Blaise M, Levacher S, Volter F. Effect of flumazenil in hepatic encephalopathy in the Effect of flumazenil in hepatic encephalopathy in the alcoholic. Electroencephalography and clinical neuro95P.

12 Cadranel JF, El Younsi M, Pidoux B, Zylberberg $P$, Behnhamon Y, Valla D, et al. Immediate improvement of hepatic encephalopathy (portal systemic encephalopathy) hepatic encephalopathy (portal systemic encephalopathy) in cirrhotic patients by flumazenil. Results of a double-
blind cross-over study. European fournal of blind cross-over study. European fou

13 Pomier-Layrargues G, Giguere JF, Lavoie J, et al. Flumazenil in cirrhotic patients in hepatic coma: a 
randomized double-blind placebo-controlled crossover trial. Hepatology 1994; 19: 32-7.

14 Mullen KD, Szauter KM, Kaminsky-Russ K. Endogenous benzodiazepine activity in physiological fluids of patients with hepatic encephalopathy. Lancet 1990: ii: 81-3.

15 Van der Rijt CCD, Schalm SW, Meulstree J, Stignen TH. Flumazenil therapy for hepatic encephalopathy, a doubleblind cross-over study. [Abstract]. Hepatology 1989; 10 (suppl): $\$ 90$.

16 Kennedy J, Parbhoo SP, MacGillivray B, Sherlock S. Effect of extracorporeal liver perfusion on the electroencephalogram of patients in coma due to acute liver failure. $Q \mathcal{F}$ Med 1973; 42: 549-61.
17 Van der Rijt CD, Schalm SW, De Groot GH, De Vlieger M. Objective measurement of hepatic encephalopathy by means of automated EEG analysis. Electroencephalogr Clin Neurophysiol 1984; 57: 423-6.

18 Pugh RN, Murray-Lyon IM, Dawson JL, Pietroni MC Williams R. Transection of the oesophagus for bleeding oesophageal varices. Br f Surg 1973; 60: 646-9.

19 Hettmansperger TP. Statistical inference based on ranks. New York: John Wiley, 1984: 133-40.

20 Basile AS, Harrison PM, Hughes RD, Gu Zi-Quang, Pannell L, McKinney A, et al. Relationship between plasma benzodiazepine receptor ligand concentrations and severity of hepatic encephalopathy. Hepatology 1994; 19: 112-21. 